\title{
Transverse Spin Structure of the Nucleon from COMPASS
}

\author{
C. Schill on behalf of the COMPASS collaboration \\ Physikalisches Institut der Albert-Ludwigs-Universität Freiburg \\ Hermann-Herder Str. 3, 79104 Freiburg, Germany.
}

The measurement of transverse spin effects in semi-inclusive deep-inelastic scattering is an important part of the COMPASS physics program. In the years 2002-2004 data were taken by scattering a $160 \mathrm{GeV}$ muon beam on a transversely polarized deuteron target. In 2007, additional data were collected on a transversely polarized proton target. New results for the Collins and Sivers asymmetries from the analysis of the proton data are presented.

\section{Introduction}

Single spin asymmetries in semi-inclusive deepinelastic scattering (SIDIS) off transversely polarized nucleon targets have been under intense experimental investigation over the past few years [12]3. They provide new insights into QCD and the nucleon structure. For instance, they allow the determination of the third yet-unknown leading-twist quark distribution function $\Delta_{T} q(x)$, the transversity distribution [45]. Additionally, they give insight into the parton transverse momentum distribution and angular momentum [6].

\section{The COMPASS experiment}

COMPASS is a fixed target experiment at the CERN SPS accelerator with a wide physics program focused on the nucleon spin structure and on hadron spectroscopy. COMPASS investigates transversity and the transverse momentum structure of the nucleon in semi-inclusive deepinelastic scattering. A $160 \mathrm{GeV}$ muon beam is scattered on a transversely polarized hydrogen or deuterium target. The scattered muon and the produced hadrons are detected in a wideacceptance two-stage spectrometer with excellent particle identification capabilities [7. In the years 2002, 2003, and 2004, data were collected on a transversely polarized ${ }^{6} \mathrm{LiD}$ target. In the run 2007 data were taken with a transversely polarized $\mathrm{NH}_{3}$ target. In the following I will focus on the new results from the data taken on the proton.

\section{The Collins asymmetry}

In semi-inclusive deep-inelastic scattering the transversity distribution $\Delta_{T} q(x)$ can be measured in combination with the chiral odd Collins fragmentation function $\Delta_{0}^{T} D_{q}^{h}(x)$. According to Collins, the fragmentation of a transversely polarized quark into an unpolarized hadron generates an azimuthal modulation of the hadron distribution with respect to the lepton scattering plane [4. The hadron yield $N\left(\Phi_{C}\right)$ can be written as:

$N\left(\Phi_{C}\right)=N_{0} \cdot\left(1+f \cdot P_{t} \cdot D_{N N} \cdot A_{C} \cdot \sin \Phi_{C}\right),(1)$

where $N_{0}$ is the average hadron yield, $f$ the fraction of polarized material in the target, $P_{t}$ the target polarization, and $D_{N N}=(1-y)\left(1-y+y^{2} / 2\right)$ the depolarization factor. The angle $\Phi_{C}$ is the Collins angle. It is defined as $\Phi_{C}=\Phi_{h}+\Phi_{s}-$ $\pi$, the sum of the hadron azimuthal angle $\Phi_{h}$ and the target spin vector azimuthal angle $\Phi_{s}$, both with respect to the lepton scattering plane 8. The measured Collins asymmetry $A_{C}$ can be factorized into a convolution of the transversity distribution $\Delta_{T} q(x)$ of quarks of flavor $q$ and the Collins fragmentation function $\Delta_{0}^{T} D_{q}^{h}(x)$, summed over all quark flavors $q$ :

$A_{C}=\frac{\Sigma_{q} e_{q}^{2} \Delta_{T} q(x) \Delta_{T}^{0} D_{q}^{h}\left(z, p_{T}^{h}\right)}{\Sigma_{q} e_{q}^{2} q(x) D_{q}^{h}\left(z, p_{T}^{h}\right)}$.

Here, $e_{q}$ is the quark charge, $D_{q}^{h}\left(z, p_{T}^{h}\right)$ the unpolarized fragmentation function, $z=E_{h} /\left(E_{\mu}-\right.$ $E_{\mu^{\prime}}$ ) the fraction of available energy carried by the hadron and $p_{T}^{h}$ the hadron transverse momentum with respect to the virtual photon direction. 
As can be seen from equation 1, the Collins asymmetry shows as a $\sin \Phi_{C}$ modulation in the number of produced hadrons.

\section{The Sivers asymmetry}

Another source of azimuthal asymmetry is related to the Sivers effect. The Sivers asymmetry rises from a coupling of the intrinsic transverse momentum $\vec{k}_{T}$ of unpolarized quarks with the spin of a transversely polarized nucleon [9. The correlation between the transverse nucleon spin and the transverse quark momentum is described by the Sivers distribution function $\Delta_{0}^{T} q\left(x, \vec{k}_{T}\right)$. The Sivers effect leads to an azimuthal modulation of the produced hadrons

$N\left(\Phi_{S}\right)=N_{0} \cdot\left(1+f \cdot P_{t} \cdot A_{S} \cdot \sin \Phi_{S}\right)$.

The Sivers angle is defined as $\Phi_{S}=\Phi_{h}-\Phi_{s}$. The measured Sivers asymmetry $A_{S}$ can be factorized into a product of the Sivers distribution function and the unpolarized fragmentation function $D_{q}^{h}(z)$ :

$A_{S}=\frac{\Sigma_{q} e_{q}^{2} \Delta_{0}^{T} q\left(x, \vec{k}_{T}\right) D_{q}^{h}(z)}{\Sigma_{q} e_{q}^{2} q(x) D_{q}^{h}(z)}$.

In this case the asymmetry $A_{S}$ shows up as the amplitude of a $\sin \Phi_{S}$ modulation in the number of produced hadrons.

Since the Collins and Sivers asymmetries are independent azimuthal modulations of the cross section for semi-inclusive deep-inelastic scattering 810, both asymmetries can be determined experimentally from the same dataset.

\section{Data sample and event selection}

In 2007 COMPASS took data with a transversely polarized proton $\left(\mathrm{NH}_{3}\right)$ target. The target consists of three cells (upstream, central and downstream) of 30, 60 and $30 \mathrm{~cm}$ length, respectively. The upstream and downstream cell are polarized in one direction while the middle cell is polarized oppositely. The target material has a high polarization of about $90 \%$ and a dilution factor of 0.15 . The direction of the target polarization was reversed every five days. The asymmetries are analyzed using at the same time data from two time periods with opposite polarization and from the different target cells. For the results presented here, about $20 \%$ of the data collected have been analyzed. The data have been selected requiring a good stability of the spectrometer within one and between consecutive periods of data taking.

To select DIS events, kinematic cuts of the squared four momentum transfer $Q^{2}>$ $1(\mathrm{GeV} / \mathrm{c})^{2}$, the hadronic invariant mass $W>$ $5 \mathrm{GeV} / \mathrm{c}^{2}$ and the fractional energy transfer of the muon $0.1<y<0.9$ were applied. The hadron sample on which the asymmetries are computed consists of all charged hadrons originating from the reaction vertex with $p_{T}^{h}>0.1 \mathrm{GeV} / \mathrm{c}$ and $z>0.2$.

The Collins and Sivers asymmetries were evaluated as a function of $x, z$, and $p_{T}^{h}$ integrating over the other two variables. The method used for the extraction is based on a two-dimensional binning in $\Phi_{h}$ and $\Phi_{s}$. The extraction of the amplitudes is then performed fitting the expression for the transverse polarization dependent part of the semi-inclusive DIS cross section [10] to the measured count rates in the target cells by a maximum likelihood method, taking into account the spectrometer acceptance. The results have been checked by several other methods described in Ref. [1.

A number of systematic studies have been performed to check against systematic effects. The systematic errors have been estimated to be 0.3 . $\sigma_{\text {stat }}$ for the Collins and $0.5 \cdot \sigma_{\text {stat }}$ for the Sivers asymmetries.

\section{Results}

In Fig. 1 the results for the Collins asymmetry on a proton target are shown as a function of $x$, $z$, and $p_{T}$ for positive and negative unidentified hadrons. For small $x$ up to $x=0.05$ the measured asymmetry is small and statistically compatible with zero, while in the last points a signal is visible. The asymmetry increases up to about $10 \%$ with opposite sign for negative and positive hadrons. Binned in $z$ and $p_{T}$ the asymmetry is compatible with zero, since most of the statistical sample is in the low $x$ region. Requiring $x>0.05$, the asymmetry signal becomes more evident both 
COMPASS 2007 proton data (part)

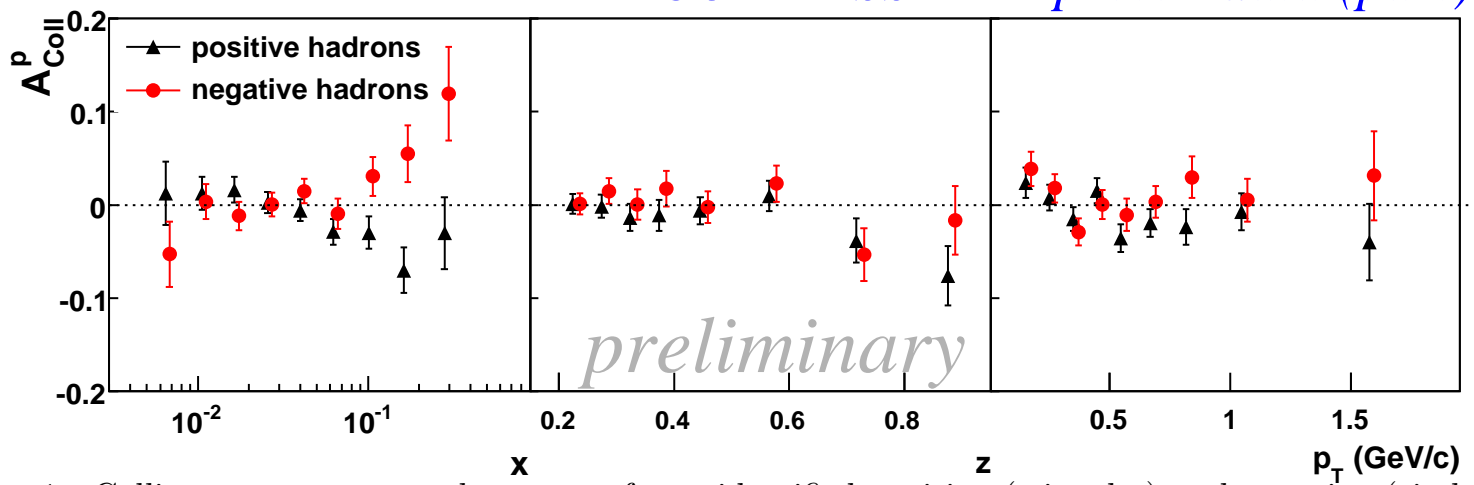

Figure 1. Collins asymmetry on the proton for unidentified positive (triangles) and negative (circles) hadrons as a function of $x, z$, and $p_{T}^{h}$.

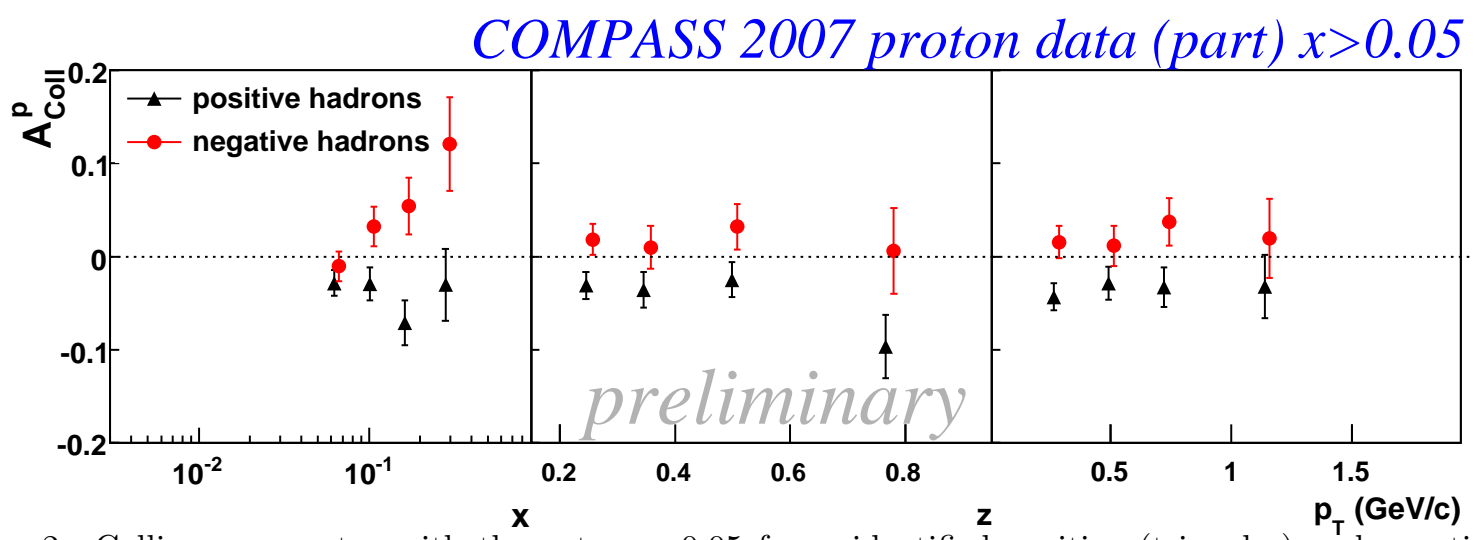

Figure 2. Collins asymmetry with the cut $x>0.05$ for unidentified positive (triangles) and negative (circles) hadrons as a function of $x, z$, and $p_{T}^{h}$.

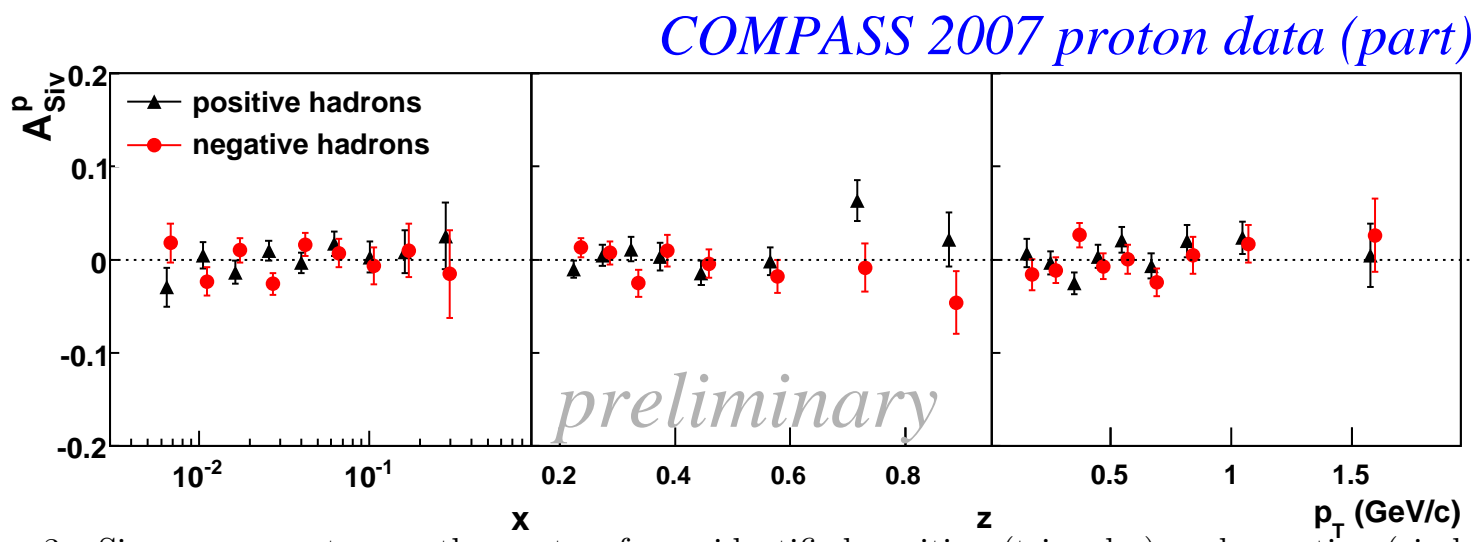

Figure 3. Sivers asymmetry on the proton for unidentified positive (triangles) and negative (circles) hadrons as a function of $x, z$, and $p_{T}^{h}$. 


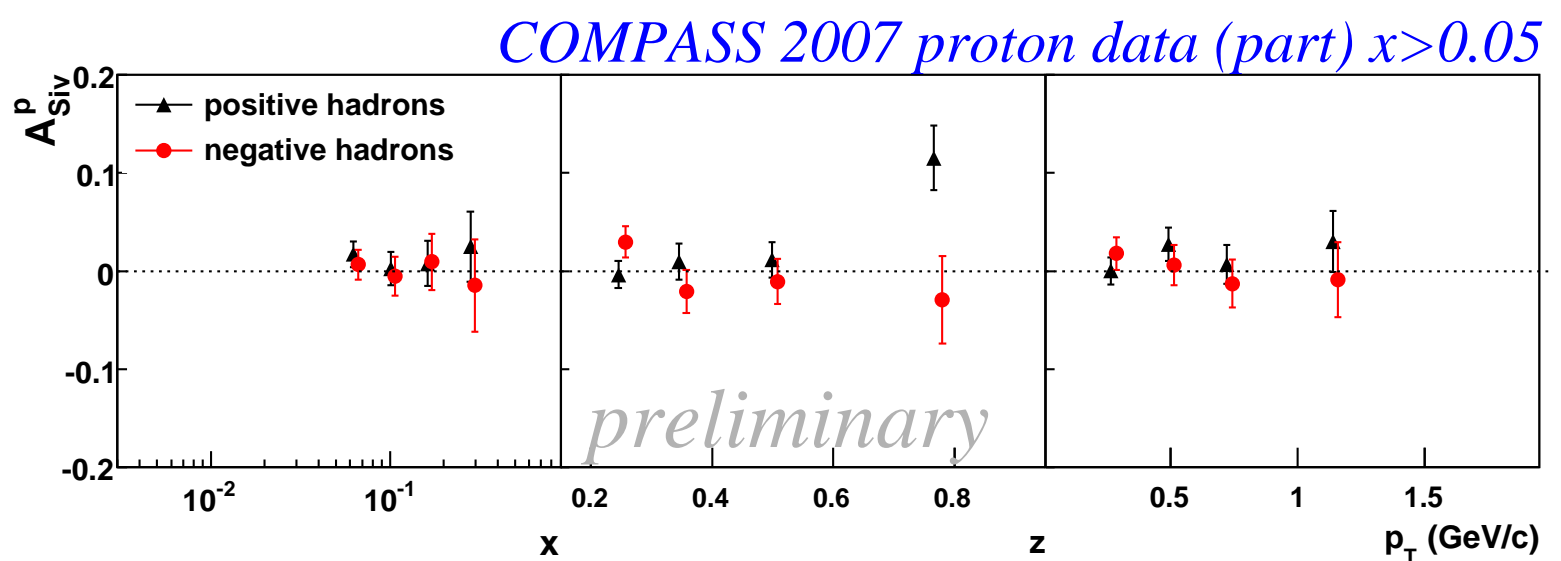

Figure 4. Sivers asymmetry with the cut $x>0.05$ for unidentified positive (triangles) and negative (circles) hadrons as a function of $x, z$, and $p_{T}^{h}$.

in $z$ and in $p_{T}^{h}$ as shown in Fig. 2. There is no appreciable $z$ or $p_{T}^{h}$ dependence.

In Fig. 3 the results for the Sivers asymmetry are shown as a function of $x, z$, and $p_{T}^{h}$. The Sivers asymmetry is small and statistically compatible with zero for both positive and negative hadrons in all the measured $x$ range. The result for positive hadrons is in contrast to the measurement done by the HERMES collaboration [11, and the two data samples are only marginally compatible. Cutting at $x>0.05$ the measured Sivers asymmetry on the proton is still compatible with zero within its statistical errors, as shown in Fig. 4.

\section{Summary}

New preliminary results for Collins and Sivers asymmetries measured at COMPASS in semiinclusive DIS on a transversely polarized proton target have been presented. For $x>0.05$, a Collins asymmetry different from zero and with opposite sign for positive and negative hadrons has been observed. Within statistical precision of the measurement, the Sivers asymmetry is compatible with zero, both for negative and positive hadrons. The dataset analyzed corresponds to about $20 \%$ of the data recorded in 2007 .

\section{REFERENCES}

1. V.Yu. Alexakhin et al. [COMPASS collaboration] Phys. Rev. Lett. 94, 202002 (2005) and E.S. Ageev et al. [COMPASS collaboration] Nucl. Phys. B765, 31 (2007), and references therein.

2. A. Airpetian et al. [HERMES Collaboration], Phys. Rev. Lett. 94, 012002 (2005).

3. A. Bacchetta and M. Radici, Phys. Rev. D67, 094002 (2003), Phys. Rev. D69, 074026 (2004) and Phys. Rev. D74, 114007 (2006).

4. J.C. Collins et al., Nucl. Phys. B420, 565 (1994).

5. X. Artru and J.C. Collins, Z. Phys. C69, 277 (1996).

6. R.L. Jaffe et al., Phys. Rev. Lett. 80, 1166 (1998).

7. P. Abbon et al. [COMPASS collaboration], NIM A577, 455-518 (2007).

8. X. Artru, hep-ph/0207309 (2002).

9. D.W. Sivers, Phys. Rev. D41, 83 (1991).

10. D. Boer and P.J. Mulders, Phys. Rev. D 57, 5780 (1998).

11. M. Diefentaler [HERMES Collaboration], proceedings of DIS2007, page 579, arXiv:0706.2242 [hep-ex]. 